ELECTRONIC RESEARCH ANNOUNCEMENTS OF THE AMERICAN MATHEMATICAL SOCIETY

Volume 5, Pages 24-34 (April 1, 1999)

S $1079-6762(99) 00056-6$

\title{
ON QUANTUM DE RHAM COHOMOLOGY THEORY
}

\author{
HUAI-DONG CAO AND JIAN ZHOU
}

(Communicated by Richard Schoen)

\begin{abstract}
We define the quantum exterior product $\wedge_{h}$ and quantum exterior differential $d_{h}$ on Poisson manifolds. The quantum de Rham cohomology, which is a deformation quantization of the de Rham cohomology, is defined as the cohomology of $d_{h}$. We also define the quantum Dolbeault cohomology. A version of quantum integral on symplectic manifolds is considered and the corresponding quantum Stokes theorem is stated. We also derive the quantum hard Lefschetz theorem. By replacing $d$ by $d_{h}$ and $\wedge$ by $\wedge_{h}$ in the usual definitions, we define many quantum analogues of important objects in differential geometry, e.g. quantum curvature. The quantum characteristic classes are then studied along the lines of the classical Chern-Weil theory. The quantum equivariant de Rham cohomology is defined in the similar fashion.
\end{abstract}

In this note we announce a construction of a deformation of the de Rham complex for any Poisson manifold. In the case of a closed symplectic manifold, its cohomology provides a deformation of the ring structure on the de Rham cohomology. More precisely, on any Poisson manifold, we define a quantum exterior product $\wedge_{h}$ of exterior forms, and quantum exterior differential $d_{h}$, such that $d_{h}^{2}=0$, and $d_{h}$ is a derivation for $\wedge_{h}$. Here $h$ is an indeterminate. We define the quantum de Rham cohomology as the cohomology of $d_{h}$. Since $d_{h}$ is a derivation with respect to $\wedge_{h}$, there is an induced quantum multiplication on the quantum de Rham cohomology.

This work grew out of our attempt to find a new way to define quantum cohomology, which has recently attracted much attention (see Tian [13] for a survey on this topic, and the introduction of Li-Tian [11] for more recent development). Intuitively, quantum cohomology provides a deformation of the ring structure on the vector space which underlies the de Rham cohomology by counting pseudoholomorphic curves in symplectic manifolds or stable curves in algebraic manifolds. Unlike many cohomology theories in algebraic topology, it is not defined as the cohomology of a graded differential algebra. For the sake of being consistent with other cohomology theories, it would be desirable to be able to do so, even though the applications of quantum cohomology do not really require this property. This is the first motivation for our construction of quantum de Rham cohomology. On the other hand, from the point of view of finding deformations of the ring structure on the de Rham cohomology per se, it is also interesting to see whether deformations of the de Rham complex can provide new deformations of the de Rham cohomology. This is the second motivation to our construction.

Received by the editors May 07, 1998.

1991 Mathematics Subject Classification. Primary 53C15, 58A12, 81R05.

Authors' research was supported in part by NSF grants DMS-96-32028 and DMS-95-04925.

(C)1999 American Mathematical Society 
Simple examples, such as flat tori and complex projective spaces, show that the quantum de Rham cohomology does give nontrivial deformation of the ring structure on the vector space underlying the de Rham cohomology. However, such examples also show that it is different from the quantum cohomology. Nevertheless, they show that the quantum de Rham cohomology provides new deformations of the de Rham cohomology: e.g. for a symplectic flat torus, the quantum cohomology does not give non-trivial deformations, while the quantum de Rham cohomology does. It is conceivable that the quantum de Rham cohomology turns out to be different from the quantum cohomology, since it does not have "strings" in it. We speculate that a version of quantum de Rham cohomology on the loop space might yield the quantum cohomology, based on the description of quantum cohomology by Vafa [14].

While it is disappointing that we cannot obtain an easier definition of quantum cohomology along the lines of ordinary de Rham cohomology this way, in retrospect, the significance of our work is that it provides a very simple version of quantum differential geometry, in the sense that our objects contain a parameter $h$, which give us the classical objects in differential geometry when $h=0$. For example, by replacing $d$ by $d_{h}$ and $\wedge$ by $\wedge_{h}$ in the usual definitions, we define many quantum analogues of important objects, such as quantum curvature and quantum characteristic classes, in differential geometry. We believe this kind of quantum differential geometry should be useful in formulating quantum theories in physics. Notice that this is quite different from Connes' non-commutative geometry. We are in the process of investigating a relationship between the two.

The definition of the quantum exterior product $\wedge_{h}$ is motivated by the MoyalWeyl multiplication and Clifford multiplication. For any finite dimensional vector space $V$, let $\left\{e_{1}, \cdots, e_{m}\right\}$ be a basis of $V$ and $\left\{e^{1}, \cdots, e^{m}\right\}$ the dual basis. Assume that $w=w^{i j} e_{i} \otimes e_{j} \in V \otimes V$. Then $w$ defines a multiplication $\wedge_{w}$ on $\Lambda\left(V^{*}\right)$, and a multiplication $*_{w}$ on $S\left(V^{*}\right)$, such that $e^{i} \wedge_{w} e^{j}=e^{i} \wedge e^{j}+w^{i j}, e^{i} *_{w} e^{j}=e^{i} \odot e^{j}+w^{i j}$. If $w \in S^{2}(V)$, then $\wedge_{w}$ is the Clifford multiplication. If $w \in \Lambda^{2}(V)$ is nondegenerate, $*_{w}$ is the Moyal-Weyl multiplication. If $w \in \Lambda^{2}(V)$, then $\Lambda_{w}$ is what we call the quantum exterior product. It is elementary to show that this multiplication is associative, and we get a quantum exterior algebra. We use it to obtain a quantum calculus on any Poisson manifold. In a way, quantum exterior algebra plays a role in Poisson or symplectic geometry similar to that of Clifford algebra in Riemannian geometry. Based on the success of Clifford algebra in Riemannian geometry, we expect that the quantum exterior algebra should be useful in Poisson or symplectic geometry.

Finally, let us point out some difference of our work with deformation quantizations or star product, of symplectic or Poisson manifolds. Let $M$ be a smooth manifold. It is well-known that $\mathcal{A}=C^{\infty}(M)$ cannot be deformed non-trivially by commutative algebras. But now it is known through the work of Kontsevich [8] that it is always possible to deform it by non-commutative algebras if a Poisson bivector field $w$ on $M$ is given. More precisely, there is a noncommutative multiplication * on $\mathcal{A}[[h]]$ such that $(\mathcal{A}[[h]], *)$ is an associative algebra with unit, and

$$
f * g=f g+\sum_{n \geq 1} h^{n} B_{n}(f, g)
$$


such that

$$
\lim _{h \rightarrow 0} \frac{f * g-g * f}{h}=\{f, g\}
$$

where $\{\cdot, \cdot\}$ is the Poisson bracket defined by $w$. For some earlier results on star products on symplectic manifolds, see Bayen et al [1], De Wilde-Lecomte [5], Fedosov [6]. In general a $\mathbb{Z}$-graded commutative algebra may have a deformation by $\mathbb{Z}_{2}$-graded commutative algebras, e.g. the quantum cohomology of $\mathbb{C P}_{n}$. Our quantum exterior product $\wedge_{h}$ defines a $\mathbb{Z}_{2}$-graded commutative, associative multiplication on $\Omega^{*}(M)[h]$, and is trivial on $\Omega^{0}(M)=C^{\infty}(M)$. (It becomes $\mathbb{Z}$-graded commutatively if we regard $h$ as an element of grade 2.)

We will present the main results of our work below. Their proofs will appear elsewhere [4].

\section{Quantum exterior Algebra}

Let $V$ be a finite dimensional vector space over a field $\mathbf{k}$ of characteristic zero, and $\Lambda\left(V^{*}\right)$ the exterior algebra generated by the dual vector space $V^{*}$. For any $v \in V$ and $\alpha \in \Lambda^{k}\left(V^{*}\right)$, denote

$$
\begin{aligned}
& (v \vdash \alpha)\left(v_{1}, \cdots, v_{k-1}\right)=\alpha\left(v, v_{1}, \cdots, v_{k-1}\right), \\
& (\alpha \dashv v)\left(v_{1}, \cdots, v_{k-1}\right)=\alpha\left(v_{1}, \cdots, v_{k-1}, v\right),
\end{aligned}
$$

for $v_{1}, \cdots, v_{k-1} \in V$. Let $\Lambda_{h}\left(V^{*}\right)=\Lambda\left(V^{*}\right)[h]=\Lambda\left(V^{*}\right) \otimes_{\mathbf{k}} \mathbf{k}[h]$. For any $w \in \Lambda^{2}(V)$, with $w=\sum_{i, j} w^{i j} e_{i} \wedge e_{j}$ with respect to a basis $\left\{e_{1}, \cdots, e_{m}\right\}$ of $V$, we define the quantum exterior product $\wedge_{h, w}: \Lambda\left(V^{*}\right) \otimes \Lambda\left(V^{*}\right) \rightarrow \Lambda\left(V^{*}\right)[h]$ by

$$
\alpha \wedge_{h, w} \beta=\sum_{n \geq 0} \frac{h^{n}}{n !} w^{i_{1} j_{1}} \cdots w^{i_{n} j_{n}}\left(\alpha \dashv e_{i_{1}} \dashv \cdots \dashv e_{i_{n}}\right) \wedge\left(e_{j_{n}} \vdash \cdots \vdash e_{j_{1}} \vdash \beta\right),
$$

for $\alpha, \beta \in \Lambda\left(V^{*}\right)$. This definition is evidently independent of the choice of the basis $\left\{e_{1}, \cdots, e_{m}\right\}$. We extend $\Lambda_{h, w}$ as a $\mathbf{k}[h]$-module map to $\Lambda_{h}\left(V^{*}\right) \otimes_{\mathbf{k}[h]} \Lambda_{h}\left(V^{*}\right)$. When there is no confusion about $w$, we will simply write $\alpha \wedge_{h} \beta$ for $\alpha \wedge_{h, w} \beta$. We are interested in $\alpha \wedge_{w} \beta$, which is just $\alpha \wedge_{1, w} \beta$. We assign to $h$ the degree 2 . Then $\Lambda_{h}\left(V^{*}\right)$ has a natural $\mathbb{Z}$-grading. Denote by $\Lambda_{h}^{[n]}\left(V^{*}\right)$ the subspace of homogeneous elements of degree $n$; then it is clear that

$$
\Lambda_{h}^{[m]}\left(V^{*}\right) \wedge_{h} \Lambda_{h}^{[n]}\left(V^{*}\right) \subset \Lambda_{h}^{[m+n]}\left(V^{*}\right) .
$$

Theorem 1 (Cao-Zhou [4]). The quantum exterior product satisfies the following properties:

$$
\begin{aligned}
\text { Supercommutativity } & \alpha \wedge_{h} \beta=(-1)^{|\alpha||\beta|} \beta \wedge_{h} \alpha, \\
\text { Associativity } & \left(\alpha \wedge_{h} \beta\right) \wedge_{h} \gamma=\alpha \wedge_{h}\left(\beta \wedge_{h} \gamma\right),
\end{aligned}
$$

for all $\alpha, \beta, \gamma \in \Lambda_{h}\left(V^{*}\right)$. Therefore, $\left(\Lambda_{h}\left(V^{*}\right), \wedge_{h}\right)$ is a deformation quantization of the exterior algebra $\left(\Lambda\left(V^{*}\right), \wedge\right)$.

The proof of (1) is trivial. The proof of (2) is of elementary nature but nontrivial. It is proved first by brute force in the case of $\operatorname{deg}(\alpha)=1$, and then by induction on $\operatorname{deg}(\alpha)$ (see [4] for details).

We can also extend $\wedge_{h}$ to $\Lambda_{h, h^{-1}}\left(V^{*}\right)=\Lambda\left(V^{*}\right)\left[h, h^{-1}\right]=\Lambda\left(V^{*}\right) \otimes_{\mathbf{k}} \mathbf{k}\left[h, h^{-1}\right]$. 
An algebra $A$ with unit $e \in A$ over a field $\mathbf{k}$ is called a $\mathbf{k}$-Frobenius algebra if there is a nondegenerate symmetric $\mathbf{k}$-bilinear function $\langle\cdot, \cdot\rangle: A \times A \rightarrow \mathbf{k}$, such that

$$
\langle\alpha \beta, \gamma\rangle=\langle\alpha, \beta \gamma\rangle,
$$

for all $\alpha, \beta, \gamma \in A$. There is a simple way to construct a structure of Frobenius algebra on any $\mathbf{k}$-algebra $A$ with unit. Let $\phi: A \rightarrow \mathbf{k}$ be a nonzero $\mathbf{k}$-functional on $A$. Set $\langle\alpha, \beta\rangle_{\phi}=\phi(\alpha \beta)$ for $\alpha, \beta \in A$. If it is nondegerate, then $\left(A,\langle\cdot, \cdot\rangle_{\phi}\right)$ is a k-Frobenius algebra. Conversely, given any Frobenius algebra $(A,\langle\cdot, \cdot\rangle)$, let $\phi(\alpha)=\langle\alpha, e\rangle$, for $\alpha \in A$; then $\langle\cdot, \cdot\rangle=\langle\cdot, \cdot\rangle_{\phi}$. Now on $\Lambda\left(V^{*}\right)$, consider a Berezin integral $\int: \Lambda\left(V^{*}\right) \rightarrow \mathbf{k}$ ( i.e., a $\mathbf{k}$-linear functional which is only nonzero on $\left.\Lambda^{\text {top }}\left(V^{*}\right)\right)$. Then it is clear that $\langle\alpha, \beta\rangle=\int \alpha \wedge_{w} \beta$ defines a structure of Frobenius algebra on $\left(\Lambda\left(V^{*}\right), \wedge_{w}\right)$.

Example 1. Let $V$ be a two dimensional vector space with a basis $\left\{e_{1}, e_{2}\right\}$, and dual basis $\left\{e^{1}, e^{2}\right\}$. Let $w=e_{1} \wedge e_{2}$. Then we have

$$
\begin{aligned}
& e^{1} \wedge_{h} e^{2}=e^{1} \wedge e^{2}+h, \\
& e^{1} \wedge_{h}\left(e^{1} \wedge e^{2}\right)=-h e^{1}, \\
& e^{2} \wedge_{h}\left(e^{1} \wedge e^{2}\right)=-h e^{2}, \\
& \left(e^{1} \wedge e^{2}\right) \wedge_{h}\left(e^{1} \wedge e^{2}\right)=-2 h e^{1} \wedge e^{2}-h^{2} .
\end{aligned}
$$

It is a tedious but straightforward exercise to check the associativity.

Example 2. Let $V$ be a $2 n$-dimensional vector space with a basis $\left\{e_{1}, \cdots, e_{2 n}\right\}$ and dual basis $\left\{e^{1}, \cdots, e^{2 n}\right\}$. Let $w=e_{1} \wedge e_{2}+\cdots+e_{2 n-1} \wedge e_{2 n}$, and $\omega=e^{1} \wedge e^{2}+$ $\cdots+e^{2 n-1} \wedge e^{2 n}$. Set

$$
\omega_{h}=e^{1} \wedge_{h} e^{2}+\cdots+e^{2 n-1} \wedge_{h} e^{2 n}=e^{1} \wedge e^{2}+\cdots+e^{2 n-1} \wedge e^{2 n}+n h .
$$

Then clearly we have

$$
\left(\omega_{h}\right)_{h}^{n+1}:=\underbrace{\omega_{h} \wedge_{h} \cdots \wedge_{h} \omega_{h}}_{n \text { times }}=0 .
$$

In particular, when $n=1$, we get $(\omega+h) \wedge_{h}(\omega+h)=0$, hence

$$
\omega \wedge_{h} \omega=-2 h \omega-h^{2},
$$

which recovers the last formula in Example 1.

\section{Quantum de Rham complex}

Let $(P, w)$ be a Poisson manifold, with bivector field $w$, whose Schouten-Nijenhuis bracket vanishes. (See Vaisman [15] for definitions.) The fiberwise quantum exterior multiplication defines

$$
\begin{gathered}
\wedge_{h}: \Omega_{h}(M) \otimes \Omega_{h}(M) \rightarrow \Omega_{h}(M), \\
\wedge_{h}: \Omega_{h, h^{-1}}(M) \otimes \Omega_{h, h^{-1}}(M) \rightarrow \Omega_{h, h^{-1}}(M),
\end{gathered}
$$

where $\Omega_{h}(M)=\Omega(M)[h]=\Omega(M) \otimes_{\mathbf{k}} \mathbf{k}[h]$ and $\Omega_{h, h^{-1}}(M)=\Omega(M)\left[h, h^{-1}\right]=$ $\Omega(M) \otimes_{\mathbf{k}} \mathbf{k}\left[h, h^{-1}\right]$. Koszul [9] defined an operator $\delta: \Omega^{k}(M) \rightarrow \Omega^{k-1}(M)$ by

$$
\delta \alpha=w \vdash d \alpha-d(w \vdash \alpha),
$$

for $\alpha \in \Omega^{k}(M)$. He also showed that $\delta^{2}=0, d \delta+\delta d=0$. We define the quantum exterior differential $d_{h}=d-(h / 2) \delta: \Omega(M)[h] \rightarrow \Omega(M)[h]$, and similarly on 
$\Omega(M)\left[h, h^{-1}\right]$. Then it is easy to see that $d_{h}^{2}=0$. One of the technical results in Cao-Zhou [4] is the following

Theorem 2. For any Poisson manifold $(M, w), d_{h}$ satisfies

$$
d_{h}\left(\alpha \wedge_{h} \beta\right)=\left(d_{h} \alpha\right) \wedge_{h} \beta+(-1)^{|\alpha|} \alpha \wedge_{h}\left(d_{h} \beta\right)
$$

for $\alpha, \beta$ in $\Omega(M)[h]$, or $\alpha, \beta$ in $\Omega(M)\left[h, h^{-1}\right]$.

This is proved first for $\operatorname{deg}(\alpha)=1$ by brute force, then by induction on $\operatorname{deg}(\alpha)$. For regular Poisson manifolds (e.g., symplectic manifolds), there is an easier proof. On such Poisson manifolds, there always exists a torsionless connection $\nabla$ which preserves $w$. Then for any local frame $\left\{e^{1}, \cdots, e^{n}\right\}$, and $\alpha \in \Omega(M)$, we have

$$
d_{h} \alpha=e^{i} \wedge_{h} \nabla_{e_{i}} \alpha .
$$

This is the analogue of a similar expression for $d+d^{*}$ in Riemannian geometry (Lawson-Michelsohn [10], Lemma II.5.13). Using the analogue of normal coordinates, the proof of Theorem 2 reduces to the associativity of the quantum exterior multiplication.

\section{Quantum de Rham cohomology}

For any Poisson manifold $(M, w)$, the (polynomial) quantum de Rham cohomology is defined by

$$
Q_{h} H_{d R}^{*}(M)=\operatorname{Ker} d_{h} / \operatorname{Im} d_{h},
$$

for the quantum exterior differential $d_{h}: \Omega(M)[h] \rightarrow \Omega(M)[h]$. The Laurent quantum de Rham cohomology is

$$
Q_{h, h^{-1}} H_{d R}^{*}(M)=\operatorname{Ker} d_{h} / \operatorname{Im} d_{h},
$$

for $d_{h}: \Omega(M)\left[h, h^{-1}\right] \rightarrow \Omega(M)\left[h, h^{-1}\right]$. As a consequence of Theorem 1 and Theorem 2 , we have

Theorem 3. The quantum de Rham cohomology $Q_{h} H_{d R}^{*}(M)$ of a Poison manifold has the following properties:

$$
\begin{aligned}
\alpha \wedge_{h} \beta & =(-1)^{|\alpha||\beta|} \beta \wedge_{h} \alpha, \\
\left(\alpha \wedge_{h} \beta\right) \wedge_{h} \gamma & =\alpha \wedge_{h}\left(\beta \wedge_{h} \gamma\right),
\end{aligned}
$$

for $\alpha, \beta, \gamma \in Q_{h} H_{d R}^{*}(M)$. Similar results hold for the Laurent quantum de Rham cohomology.

The complex $\left(\Omega(M)[h], d_{h}\right)$ can be regarded as a double complex $\left(C^{p, q},-h \delta / 2, d\right)$, where $C^{p, q}=h^{p} \Omega^{q-p}(M), p \geq 0$. This is the analogue of Brylinski's double complex $\mathcal{C}_{. .}(M)([3], \S 1.3)$. By the standard theory for a double complex (Bott-Tu [2], §14), there are two spectral sequences $E$ and $E^{\prime}$ abutting to $H^{*}\left(\Omega[h], d_{h}\right)=$ $Q_{h} H_{d R}^{*}(M)$, with $E_{1}^{p, q}=h^{p} H^{q}\left(C^{p, *}, d\right)=h^{p} H_{d R}^{q-p}(M),\left(E_{1}^{\prime}\right)^{p, q}=h^{p} H^{*}\left(C^{*, q}, \delta\right)=$ $h^{p} \mathrm{PH}_{q-p}(M), p \geq 0$. Since nontrivial $E_{1}^{p, q}$ all have $p+q$ even, and the differential $d_{r}$ changes the parity of $p+q$, it is routine to prove the following

Theorem 4. For a Poisson manifold with odd Betti numbers all vanishing, the spectral sequence $E$ degenerates at $E_{1}$, i.e. $d_{r}=0$ for all $r \geq 0$. Hence $Q_{h} H_{d R}^{*}(M)$ is a deformation quantization of $H_{d R}^{*}(M)$. 
Brylinski [3] proved that on a closed Kähler manifold $(M, \omega)$, every de Rham cohomology class has a representative $\alpha$ such that $d \alpha=0, \delta \alpha=0$. This implies the following

Theorem 5. For a closed Kähler manifold $M$, the spectral sequence $E$ degenerates at $E_{1}$, i.e. $d_{r}=0$ for all $r \geq 0$. Hence $Q_{h} H_{d R}^{*}(M)$ is a deformation quantization of $H_{d R}^{*}(M)$.

Similarly, we regard $\left(\Omega(M)\left[h, h^{-1}\right], d-h \delta / 2\right)$ as a double complex $\left(\widetilde{C}^{p, q},-h \delta / 2, d\right)$, where $\widetilde{C}^{p, q}=h^{p} \Omega^{q-p}(M), p, q \in \mathbb{Z}$. This is essentially Brylinski's double complex

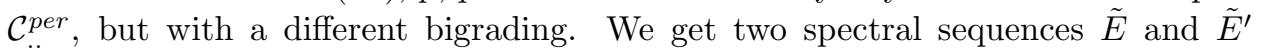
abutting to $Q_{h, h^{-1}} H_{d R}^{*}(M)$, with $\tilde{E}_{1}^{p, q}=h^{p} H_{d R}^{q-p}(M),\left(\tilde{E}_{1}^{\prime}\right)^{p, q}=h^{p} H^{*}\left(C^{*, q}, \delta\right)=$ $h^{p} H_{q-p}(M), p, q \in \mathbb{Z}$. It is clear that an analogue of Theorem 4 holds for $\tilde{E}$. On the other hand, by a method of Brylinski [3], one can prove the following

Theorem 6. For any compact symplectic manifold without boundary, the spectral sequences $\tilde{E}$ and $\tilde{E}^{\prime}$ degenerate at $\tilde{E}_{1}$ and $\tilde{E}_{1}^{\prime}$, respectively. Hence $Q_{h, h^{-1}} H_{d R}^{*}(M)$ is a Laurent deformation quantization of $H_{d R}^{*}(M)$.

Fixing an isomorphism $H_{d R}^{2 n}(M) \cong \mathbb{R}$ then defines a structure of Frobenius algebra on $\left(H_{d R}^{*}(M), \wedge_{w}\right)$.

Remark. It would be interesting to know whether Theorems 4-6 hold for regular Poisson manifolds, or even general Poisson manifolds.

\section{Some examples}

Our first example is the flat symplectic torus $(T, \omega)$. By virtue of Theorem 6 , $Q_{h, h^{-1}} H_{d R}^{*}(T)$ is isomorphic to $H^{*}(T) \otimes \mathbb{R}\left[h, h^{-1}\right]$. Picking up a flat Riemannian metric on $T$ which is compatible with $\omega$, we can represent de Rham cohomology classes by harmonic forms on $T$. But all these forms are parallel, hence, by (4), $d_{h}$-closed. The quantum exterior product of any two such forms can be found by restricting to a point on the torus. Therefore, the ring structure on quantum de Rham cohomology is the quantum exterior algebra for the tangent space of any point on the torus. The case of a 2-torus can be explicitly described by Example 1. Notice that since the second homotopy group of a torus is trivial, there is no nontrivial pseudo-holomorphic $S^{2}$ in a torus. Hence the quantum cohomology of a symplectic torus is trivial. But the quantum de Rham cohomology is obviously nontrivial.

Similarly, for a complex projective space $\mathbb{C P}_{n}$ with standard Kähler structure, both ordinary and quantum de Rham cohomology is generated by the symplectic form $\omega$. Again since harmonic forms are parallel, we can reduce the calculation to the tangent space of a point, which is a symplectic vector space. Then the result of Example 2 can be used. It is clear now that the quantum cohomology and quantum de Rham cohomology produce different deformations: e.g. on $\mathbb{C P}_{1}$, the quantum cohomology is

$$
\mathbb{R}[\omega][q] /\left(\omega^{2}-q\right),
$$

while the quantum de Rham cohomology is

$$
\mathbb{R}[\omega][h] /\left(\omega^{2}+2 h \omega+h^{2}\right) .
$$




\section{Quantum Hard Lefschetz Theorem}

For a closed symplectic manifold, the analogue of the Hard Lefschetz Theorem (see [7]) holds for $Q_{h, h^{-1}} H_{d R}^{*}(M)$. We begin with a $2 n$-dimensional symplectic vector space $(V, \omega)$. Brylinski [3] defined a symplectic star operator $*: \Lambda^{k}\left(V^{*}\right) \rightarrow$ $\Lambda^{2 n-k}\left(V^{*}\right)$. We can extend it to $\Lambda_{h, h^{-1}}$ by setting $* h=h^{-1}$, and $* h^{-1}=h$. Define $L_{h}: \Lambda_{h, h^{-1}}\left(V^{*}\right) \rightarrow \Lambda_{h, h^{-1}}\left(V^{*}\right)$ by $L_{h}(\alpha)=\omega \wedge_{h} \alpha$. Define $L_{h}^{*}=-* L *$, and $A_{h}: \Lambda_{h, h^{-1}}\left(V^{*}\right) \rightarrow \Lambda_{h, h^{-1}}\left(V^{*}\right)$ by $A_{h}(\alpha)=(n-k) \alpha$, for $\alpha \in \Lambda_{h, h^{-1}}^{[k]}\left(V^{*}\right)$. Then we have

Lemma 1. The following identities hold:

$$
\left[L_{h}, L_{h}^{*}\right]=0, \quad\left[L_{h}, A_{h}\right]=2 L_{h}, \quad\left[L_{h}^{*}, A_{h}\right]=-2 L_{h}^{*} .
$$

Furthermore, if we regard multiplications by $h$ and $h^{-1}$ as operators, then we have

$$
\left[h, h^{-1}\right]=0, \quad\left[L_{h}, h^{ \pm 1}\right]=\left[L_{h}^{*}, h^{ \pm 1}\right]=0, \quad\left[A_{h}, h^{ \pm}\right]= \pm 2 h^{ \pm 1} .
$$

Thus we cannot use the representation theory of $\operatorname{sl}(2, \mathbb{C})$ as in the classical theory. Notice that multiplication by $h$ is an isomorphism, whose inverse is multiplication by $h^{-1}$. Let $M_{h}=h^{-1} L_{h}, M_{h}^{*}=h L_{h}^{*}$; then $M_{h}, M_{h}^{*}, A_{h}$ form an abelian algebra. Notice that it now suffices to find the eigenvalues of $M_{h}$ on $\Lambda_{h, h^{-1}}^{[0]}\left(V^{*}\right)$ and $\Lambda_{h, h^{-1}}^{[1]}\left(V^{*}\right)$. In Cao-Zhou [4], this is done by induction on the dimension of $V$. In suitable bases, the matrix $M_{n}$ of $M_{h}$ for $V$ (of dimension $2 n$ ) can be expressed in terms of the matrix $M_{n-1}$ of $M_{h}$ for a symplectic subspace of dimension $2 n-2$. Essentially, it is of the form

$$
M_{n}=\left(\begin{array}{cccc}
M_{n-1} & -I & 0 & 0 \\
I & M_{n-1}+2 I & 0 & 0 \\
0 & 0 & M_{n-1}+I & 0 \\
0 & 0 & 0 & M_{n-1}+I
\end{array}\right) .
$$

For details, see Cao-Zhou [4]. We have the following

Lemma 2. Let $\left\{M_{n}\right\}$ be a sequence of square matrices with coefficient in $\mathbf{k}$, obtained in the following way:

$$
M_{n+1}=\left(\begin{array}{cc}
M_{n} & -I \\
I & M_{n}+2 I
\end{array}\right)
$$

for $n \geq 1$, where $I$ is the identity matrix of the same size as $M_{n}$. For any $\lambda \in \mathbf{k}$, and $n \geq 1$, we have

$$
\operatorname{det}\left(M_{n+1}+\lambda I\right)=\operatorname{det}\left[M_{n}+(\lambda+1) I\right]^{2} .
$$

Therefore, the eigenvalues of $M_{n+1}$ can be obtained by adding 1 to those of $M_{n}$, with the multiplicities doubled.

As a consequence, we find that the eigenvalues of $M_{h}$ on $\Lambda_{h, h^{-1}}\left(V^{*}\right)$ are $n$ and $n \pm \sqrt{5} / 2$, when $\operatorname{dim}(V)=2 n$. For $M_{h}^{*}$, they are $-n$ and $-n \pm \sqrt{5} / 2$. Therefore, we have

Theorem 7. For a symplectic vector space $V$, the operators $L_{h}$ and $L_{h}^{*}$ are isomorphisms. Furthermore, $\Lambda_{h, h^{-1}}\left(V^{*}\right)$ decomposes into one dimensional eigenspaces of $h^{-1} L_{h}$ (or $h L_{h}^{*}$ ) with nonzero eigenvalues. 
Lemma 3. On a symplectic manifold $(M, \omega)$, we have

$$
\left[L_{h}, d_{h}\right]=0, \quad\left[L_{h}^{*}, d_{h}\right]=0, \quad\left[A_{h}, d_{h}\right]=-d_{h} .
$$

As a consequence, we get:

Theorem 8 (Quantum Hard Lefschetz Theorem). For any symplectic manifold $\left(M^{2 n}, \omega\right)$, its Laurent quantum de Rham cohomology $Q_{h, h^{-1}} H_{d R}^{*}(M)$ decomposes into one-dimensional eigenspaces of the operator $h^{-1} L_{h}$ (or $h L_{h}^{*}$ ) with nonzero eigenvalues. In particular, $L_{h}$ and $L_{h}^{*}$ are isomorphisms.

Remark. In classical algebraic geometry, the Hard Lefschetz Theorem can be proved by considering the Lie algebra generated by the operator $L$ given by multiplication with the symplectic form $\omega$ and its adjoint $\Lambda$ by commutators. The above theorem get its name since we consider $L_{h}$ given by the quantum exterior product with $\omega$. It is not related to the symplectic version of the Hard Lefschetz Theorem proved by Mathieu [12] and Yan [16], which does not hold for all closed symplectic manifolds.

\section{Complexified QuAntum exterior Algebra}

We also consider real vector space $V$ with an almost complex structure $J \in$ $\operatorname{End}(V)$ such that $J^{2}=-I d$. There is an induced linear transformation $\Lambda^{2} J$ : $\Lambda^{2}(V) \rightarrow \Lambda^{2}(V)$. For any bivector $w \in \Lambda^{2}(V), J$ is said to preserve $w$ if $\Lambda^{2} J(w)=$ $w$. Given any bivector $w$ which is preserved by $J$, we can define the quantum exterior product on $\Lambda_{h}\left(V^{*}\right)$ as in the last section. Now if we tensor everything by $\mathbb{C}$, we get a complex algebra $\mathbb{C} \Lambda_{h}\left(V^{*}\right)$, which is a deformation quantization of $\mathbb{C} \Lambda\left(V^{*}\right):=\Lambda\left(V^{*}\right) \otimes_{\mathbb{R}} \mathbb{C}=\Lambda_{\mathbb{C}}\left(V^{*} \otimes_{\mathbb{R}} \mathbb{C}\right)$. As in complex geometry, we can exploit a natural decomposition as follows. $J$ can be uniquely extended to a complex linear endomorphism, denoted also by $J$, of $\mathbb{C} V$ also satisfying $J^{2}=-I d$. There is a natural identification of complex vector spaces $\mathbb{C} V \cong V^{1,0} \oplus V^{0,1}$, where $V^{1,0}$ and $V^{0,1}$ are eigenspaces of $J$ with eigenvalues $\sqrt{-1}$ and $-\sqrt{-1}$ respectively. As a consequence, there are decompositions

$$
\begin{aligned}
\mathbb{C} \Lambda(V) & =\bigoplus_{p, q} \Lambda^{p, q}(V), \\
\mathbb{C} \Lambda\left(V^{*}\right) & =\bigoplus_{p, q} \Lambda^{p, q}\left(V^{*}\right),
\end{aligned}
$$

where $\Lambda^{p, q}(V) \cong \Lambda_{\mathbb{C}}^{p}\left(V^{1,0}\right) \otimes_{\mathbb{C}} \Lambda_{\mathbb{C}}^{q}\left(V^{0,1}\right)$, and $\Lambda^{p, q}\left(V^{*}\right) \cong \Lambda_{\mathbb{C}}^{p}\left(\left(V^{1,0}\right)^{*}\right) \otimes_{\mathbb{C}} \Lambda_{\mathbb{C}}^{q}\left(\left(V^{0,1}\right)^{*}\right)$. We give $\mathbb{C} \Lambda_{h}\left(V^{*}\right)$ the following $\mathbb{Z} \times \mathbb{Z}$-bigrading: elements in $\Lambda^{p, q}\left(V^{*}\right)$ has bidegree $(p, q)$, and $h$ has bidegree $(1,1)$. Since $w$ is preserved by $J$, it belongs to $\Lambda^{1,1}(V)$ after complexification. Denote by $\Lambda_{h}^{[p, q]}\left(V^{*}\right)$ the space of homogeneous elements of bidegree $(p, q)$. It is then straightforward to see that

$$
\Lambda_{h}^{[p, q]}\left(V^{*}\right) \wedge_{h} \Lambda_{h}^{[r, s]}\left(V^{*}\right) \subset \Lambda_{h}^{[p+r, q+t]}\left(V^{*}\right) .
$$

Now let $\omega$ be a symplectic form on $V$ which is compatible with an almost complex structure on $V$. Namely, rank of $\omega$ is $2 n=\operatorname{dim}(V), w(J \cdot, J \cdot)=\omega(\cdot, \cdot)$, and $g(\cdot, \cdot):=$ $\omega(\cdot, J \cdot)$ is a positive definite element of $S^{2}\left(V^{*}\right)$. Then $\omega$ induces a natural Hermitian metric $H$ on $\mathbb{C} \Lambda\left(V^{*}\right)$, such that

$$
H\left(\alpha \wedge_{w} \beta, \gamma\right)=H\left(\alpha, \beta \wedge_{w} \gamma\right)
$$


for any $\alpha, \beta, \gamma \in \mathbb{C} \Lambda\left(V^{*}\right)$. Here $w \in \Lambda^{2}(V)$ is obtained from $\omega$ by "raising the indices" (for details, see Cao-Zhou [4], §1.5). This shows that the algebra $\left(\mathbb{C} \Lambda\left(V^{*}\right), \wedge_{w}\right)$ has a structure of Hermitian Frobenius algebra.

\section{Quantum Dolbeault cohomology}

On a complex manifold $(M, J)$ with a Poisson structure $w$, such that $J$ preserves $w$, we define $\delta^{-1,0}: \Omega^{p, q}(M) \rightarrow \Omega^{p-1, q}(M)$ and $\delta^{0,-1}(M): \Omega^{p, q}(M) \rightarrow \Omega^{p, q-1}(M)$ by

$$
\begin{aligned}
& \delta^{0,-1} \alpha=w \vdash(\partial \alpha)-\partial(w \vdash \alpha), \\
& \delta^{-1,0} \alpha=w \vdash(\bar{\partial} \alpha)-\bar{\partial}(w \vdash \alpha),
\end{aligned}
$$

for $\alpha \in \Omega^{p, q}(M)$. Set $\partial_{h}=\partial-(h / 2) \delta^{0,-1}$, and $\bar{\partial}_{h}=\bar{\partial}-(h / 2) \delta^{-1,0}$. Then $d_{h}=\partial_{h}+\bar{\partial}_{h}$. Now $0=d_{h}^{2}=\partial_{h}^{2}+\left(\partial_{h} \bar{\partial}_{h}+\bar{\partial}_{h} \partial_{h}\right)+\bar{\partial}_{h}^{2}$, since they have bidegrees $(2,0),(1,1)$ and $(0,2)$ respectively. Hence, we have

$$
\partial_{h}^{2}=0, \quad \partial_{h} \bar{\partial}_{h}+\bar{\partial}_{h} \partial_{h}=0, \quad \bar{\partial}_{h}^{2}=0 .
$$

We then define

$$
\begin{aligned}
Q_{h} H^{p, *}(M) & =H\left(\Omega_{h}^{[p, *]}(M), \bar{\partial}_{h}\right), \\
Q_{h, h^{-1}} H^{p, *}(M) & =H\left(\Omega_{h, h^{-1}}^{[p, *]}(M), \bar{\partial}_{h}\right) .
\end{aligned}
$$

They will be called the quantum Dolbeault cohomology and Laurent quantum Dolbeault cohomology respectively. Several relevant spectral sequences and their degeneracy are considered in Cao-Zhou [4].

\section{Quantum integral and quantum Stokes Theorem}

Let $(M, \omega)$ be a closed $2 n$-dimensional symplectic manifold. Define an integral $\int_{h}: \Omega_{h}(M) \rightarrow \mathbb{R}[h]$ as follows. For any $\alpha \in \Omega^{j}(M)$, if $j$ is odd, set $\int_{h} \alpha=0$; if $j=2 n-2 k$ for some integer $k$, set

$$
\int_{h} \alpha=\int_{M} \alpha \wedge \frac{\omega^{k}}{k !}
$$

Extend $\int_{h}$ to $\Omega_{h}(M)$ as a $\mathbb{R}[h]$-module map. We call $\int_{h}$ the quantum integral. Then we have

Theorem 9 (Quantum Stokes Theorem). For any $\alpha \in \Omega^{j}(M)$, we have $\int_{h} d \alpha=0$, $\int_{h} h \delta \alpha=0$. Therefore

$$
\int_{h} d_{h} \alpha=0 .
$$

\section{Quantum Chern-Weil theory}

Given a real or complex vector bundle $E \rightarrow M$ over a Poisson manifold $M$, and a connection $\nabla^{E}$ on it, we define the quantum covariant derivative

$$
d_{h}^{\nabla^{E}}: \Omega_{h}^{*}(E) \rightarrow \Omega_{h}^{*}(E)
$$


as follows. Let $\mathbf{s}$ be a local frame of $E$ and $\theta$ the connection 1-form in this frame. Then we have $\nabla \mathbf{s}=\mathbf{s} \otimes \theta$, i.e.,

$$
\nabla \mathbf{s}_{j}=\sum_{k=1}^{n} \mathbf{s}_{k} \otimes \theta_{j}^{k}
$$

For $\alpha=\mathbf{s} \otimes \phi$, where $\phi$ is a vector-valued form, we define

$$
d_{h}^{\nabla^{E}} \alpha=\mathbf{s} \otimes\left(\theta \wedge_{h} \phi+d_{h} \phi\right)=\sum \mathbf{s}_{k} \otimes\left(\theta_{j}^{k} \wedge_{h} \phi^{j}+d_{h} \phi^{k}\right) .
$$

It is straightforward to check that the definition of $d_{h}^{\nabla^{E}}$ is independent of the choice of the local frames (Cao-Zhou [4], Lemma 7.1). Furthermore, there is an element $R_{h}^{E} \in \Omega_{h}^{2}(\operatorname{End}(E))$ such that for each $k \geq 0,\left(d_{h}^{\nabla^{E}}\right)^{2}$ on $\Omega_{h}^{k}(M)$ is given by $\left(d_{h}^{\nabla^{E}}\right)^{2} \Phi=\Phi \wedge_{h} R_{h}^{E}$, for any $\Phi \in \Omega_{h}^{*}(E) . R_{h}^{E}$ is called the quantum curvature of $\nabla^{E}$. In a local frame, $R_{h}^{E}$ is given by

$$
F_{h}=d_{h} \theta+\theta \wedge_{h} \theta
$$

where $\theta$ is the connection 1-form matrix in the local frame.

If $p$ is a polynomial on the space of $n \times n$-matrices, such that $p\left(G^{-1} A G\right)=p(A)$, for any $n \times n$-matrix $A$, and invertible $n \times n$-matrix $G$, then $p\left(F_{h}\right)$ for different frames patch up to a well-defined element $p\left(R^{E}\right) \in \Omega^{*}(M)[h]$. Similarly to the ordinary Chern-Weil theory, it is easy to see that $d_{h} p\left(R^{E}\right)=0$. So it defines a class in $Q_{h} H_{d R}^{*}(M)$. The usual construction of transgression operator carries over to show that this class is independent of the choice of the connection $\nabla^{E}$. In this way, one can define quantum Chern classes, quantum Euler class, etc. We will call them quantum characteristic classes. It is clear that we can repeat the same story in the Laurent case.

\section{Quantum equivariant de Rham cohomology}

Let $(M, w)$ be a Poisson manifold that admits an action by a compact connected Lie group $G$, such that the $G$-action preserves the Poisson bivector field $w$. Let $\mathfrak{g}$ be the Lie algebra of $G,\left\{\xi_{a}\right\}$ a basis of $\mathfrak{g}$ and $\left\{\Theta^{a}\right\}$ the dual basis in $S^{1}\left(\mathfrak{g}^{*}\right)$. Denote by $\iota_{a}$ the contraction by the vector field generated by the one parameter group corresponding to $\xi_{a}$, and $L_{a}$ the Lie derivative by the same vector field. Imitating the Cartan model for equivariant cohomology, we consider the operator $D_{h G}=d_{h}+\Theta^{a} \iota_{a}=d-h \delta / 2+\Theta^{a} \iota_{a}$ acting on $\left(S\left(\mathfrak{g}^{*}\right) \otimes \Omega(M)\right)^{G}[h]$. It is well known that $d+\Theta^{a} \iota_{a}$ maps $\left(S\left(\mathfrak{g}^{*}\right) \otimes \Omega(M)\right)^{G}$ to itself. Since the $G$-action preserves $w$, it is easy to check that $\delta$ also preserves $\left(S\left(\mathfrak{g}^{*}\right) \otimes \Omega(M)\right)^{G}$. Therefore, $D_{h G}$ is an operator from $\left(S\left(\mathfrak{g}^{*}\right) \otimes \Omega(M)\right)^{G}[h]$ to itself. Now on $\left(S\left(\mathfrak{g}^{*}\right) \otimes \Omega(M)\right)^{G}[h]$, we have

$$
\begin{aligned}
D_{h G}^{2} & =d_{h}^{2}+\left(\Theta^{a} \iota_{a}\right)^{2}+\Theta^{a}\left(d \iota_{a}+\iota_{a} d\right)-h \Theta^{a}\left(\delta \iota_{a}+\iota_{a} \delta\right) \\
& =-h \Theta^{a}\left(\delta \iota_{a}+\iota_{a} \delta\right) .
\end{aligned}
$$

Since $\delta=\iota_{w} d-d \iota_{w}$, it is straightforward to verify that $\delta \iota_{a}+\iota_{a} \delta=0$. Hence, $D_{h G}^{2}=0$. We call the cohomology

$$
Q_{h} H_{G}^{*}(M):=H^{*}\left(\left(S\left(\mathfrak{g}^{*}\right) \otimes \Omega(M)\right)^{G}[h], D_{h G}\right)
$$

the quantum equivariant de Rham cohomology. Similar definitions can be made by using Laurent deformation. We will study the quantum equivariant de Rham cohomology in a forthcoming paper. 


\section{REFERENCES}

[1] F. Bayen, M. Flato, C. Fronsdal, A. Lichnerowicz, D. Sternheimer, Deformation theory and quantization. I. Deformations of symplectic structures, Ann. Physics 111 (1978), no. 1, 61110. MR 58:14737a

[2] R. Bott, L. W. Tu, Differential forms in algebraic topology. Graduate Texts in Mathematics, 82. Springer-Verlag, New York-Berlin, 1982. MR 83i:57016

[3] J.-L. Brylinski, A differential complex for Poisson manifolds, J. Differential Geom. 28 (1988), no. 1, 93-114. MR 89m:58006

[4] H.-D. Cao, J. Zhou, Quantum de Rham cohomology, preprint, math.DG/9806157, 1998.

[5] M. De Wilde, P. B. A. Lecomte, Existence of star-products and of formal deformations of the Poisson Lie algebra of arbitrary symplectic manifolds, Lett. Math. Phys. 7 (1983), no. 6, 487-496. MR 85j:17021

[6] B. V. Fedosov, A simple geometrical construction of deformation quantization, J. Differential Geom. 40 (1994), no. 2, 213-238. MR 95h:58062

[7] P. Griffiths, J. Harris, Principles of algebraic geometry. Pure and Applied Mathematics. Wiley-Interscience [John Wiley \& Sons], New York, 1978. MR 80b:14001

[8] M. Kontsevich, Deformation quantization of Poisson manifolds, I, preprint, q-alg/9709040.

[9] J.-L. Koszul, Crochet de Schouten-Nijenhuis et cohomologie, The mathematical heritage of Élie Cartan (Lyon, 1984). Asté'risque1985, Numero Hors Serie, 257-271. MR 88m:17013

[10] H. B. Lawson, Jr., M.-L. Michelsohn, Spin geometry. Princeton Mathematical Series, 38. Princeton University Press, Princeton, NJ, 1989. MR 91g:53001

[11] J. Li, G. Tian, Comparison of the algebraic and the symplectic Gromov-Witten invariants, preprint, December 1997, available at alg-geom/9712035.

[12] O. Mathieu, Harmonic cohomology classes of symplectic manifolds, Comment. Math. Helv. 70 (1995), no. 1, 1-9. MR 96e:58004

[13] G. Tian, Quantum cohomology and its associativity, Current developments in mathematics, 1995 (Cambridge, MA), pp. 361-401, Internat. Press, Cambridge, MA, 1994. MR 98k:58031

[14] C. Vafa, Topological mirrors and quantum rings, Essays on mirror manifolds, pp. 96-119, Internat. Press, Hong Kong, 1992. MR 94c:81193

[15] I. Vaisman, Lectures on the geometry of Poisson manifolds. Progress in Mathematics, 118, Birkhäuser, Basel, 1994. MR 95h:58057

[16] D. Yan, Hodge structure on symplectic manifolds, Adv. Math. 120 (1996), no. 1, 143-154. MR 97e:58004

Department of Mathematics, Texas A\&M University, College Station, TX 77843

E-mail address: cao@math.tamu.edu

Department of Mathematics, Texas A\&M University, College Station, TX 77843

E-mail address: zhou@math.tamu.edu 